\title{
Echinococcus multilocularis in Kyrgyzstan: similarity in the Asian EmsB genotypic profiles from village populations of Eastern mole voles (Ellobius tancrei) and dogs in the Alay valley
}

\author{
E. Afonso ${ }^{1}$, J. Knapp ${ }^{1}$, N. Tête ${ }^{1}$, G. Umhang ${ }^{2}$, D. Rieffel ${ }^{1}$, \\ F. van Kesteren ${ }^{3}$, I. Ziadinov ${ }^{4}$, P.S. Craig ${ }^{3}$, P.R. Torgerson ${ }^{4}$ \\ and P. Giraudoux ${ }^{1,5 *}$
}

${ }^{1}$ Chrono-environnement Laboratory, UMR 6249 CNRS, University of Franche-Comté, 16 route de Gray, F-25030 Besançon, France: ${ }^{2}$ ANSES, Nancy Laboratory for Rabies and Wildlife, National, Wildlife Surveillance and Eco-epidemiology Unit, Technopôle Agricole et Vétérinaire, B.P. 40009, 54220 Malzéville, France: ${ }^{3}$ Cestode Zoonoses Research Group, School of Environment and Life Sciences, University of Salford, M5 4WT Salford, UK: ${ }^{4}$ Section of Epidemiology, Vetsuisse Faculty, University of Zurich, Switzerland: ${ }^{5}$ Institut Universitaire de France, Paris, France

(Received 4 March 2015; Accepted 10 May 2015; First Published Online 3 July 2015)

\begin{abstract}
Echinococcus multilocularis is a cestode that causes human alveolar echinococcosis, a lethal zoonosis of public health concern in central Asia and western China. In the present study, one of 42 Eastern mole voles (Ellobius tancrei) caught in Sary Mogol (Alay valley, southern Kyrgyzstan) presented liver lesions with E. multilocularis from which the EmsB target was amplified. The Asian profile obtained was almost identical to one amplified from domestic dog faeces collected in a nearby village. This observation adds additional information to the potential role of E. tancrei in the transmission of E. multilocularis, and to the known distribution range of E. multilocularis (Asian strain) in central Asia.
\end{abstract}

\section{Introduction}

The taeniid cestode Echinococcus multilocularis is the causative agent of human alveolar echinococcosis (AE), a potentially lethal helminthic zoonosis (Eckert \& Deplazes, 2004). Although AE is a rare disease within the distribution range of the parasite, several endemic areas have been reported in North America, Europe and Asia (Vuitton et al., 2003). Echinococcus multilocularis has a complex life cycle that involves carnivores (principally foxes) as definitive hosts, and cricetid rodents

*E-mail: patrick.giraudoux@univ-fcomte.fr (e.g. Microtus spp.) or lagomorphs (e.g. Ochotona spp.) as intermediate hosts. Dogs are also good definitive hosts. The assemblage of wildlife host communities varies according to ecological features on multiple spatial scales (Giraudoux et al., 2006). From a genetic point of view, E. multilocularis appears as an organism with low polymorphism (Haag et al., 1997; Eckert et al., 2001). However, distinct European, Asian and North American genotypes have been described (Bretagne et al., 1996; Bart et al., 2006) and the geographical location of the transitional zone between Asian and European genotypes, somewhere between eastern Europe and western China, is currently unknown. Furthermore, a tandemly repeated microsatellite, EmsB, has been used to describe the relative diversity 
of parasite genetic profiles on both regional and local scales (Knapp et al., 2007, 2008, 2009).

Kyrgyzstan is one of the five republics of central Asia that, with northern Iran, eastern Turkey and Caucasia, provides the geographical link between the transmission foci of Asia and continental Europe. However, nothing is known about the genotypes of E. multilocularis circulating in the area, which theoretically may belong either to the Asian or the European clades, or both. In Kyrgyzstan, cystic echinococcosis caused by E. granulosus, is a national public health concern across the whole country (Torgerson et al., 2006). The highest incidences of human alveolar echinococcosis, however, are currently recorded in the sub-national administrative regions of Issyk-kul, Naryn and Osh, the latter including the Alay valley (Usubalieva et al., 2013). In the Alay valley (altitude 2900-3500 m) land cover is mostly Alpine grassland. Echinococcus multilocularis definitive hosts are the red fox (Vulpes vulpes) and domestic dogs (Ziadinov et al., 2008, 2010). In terms of potential prey biomass, the three dominant species in local small mammal assemblages are: Microtus gregalis (the narrow-headed vole), Cricetulus migratorius (the grey dwarf hamster) and Ellobius tancrei, (the Eastern mole vole) (Giraudoux et al., 2013 and unpublished). Although, historically, M. gregalis and E. tancrei have been found to be infected naturally in Kyrgyzstan (Gagarin et al., 1957; Tokobaev, 1959), their relative contribution to E. multilocularis transmission is still unknown. Ellobius tancrei has a wide distribution range, stretching from north-eastern Turkmenistan and eastern Uzbekistan through China and Mongolia (Batsaikhan \& Tinnin, 2008). More than 50 years ago this species was already recorded as being infected naturally with E. multilocularis in Kyrgyzstan (Tokobaev, 1959), but in the original paper it was likely confused with E. talpinus, the Northern mole vole, which actually is not present in Kyrgyztan. No other mention since then of E. tancrei voles infected by E. multilocularis could be found in the literature. However, population surges of this species have been observed regularly, for instance in the Alay valley, the Tien Shan (Narati area, Xinjiang, China) and the Altai Mountains (Giraudoux et al., 2008, 2013 and unpublished).

Here we report infection of E. tancrei in Sary Mogol village $\left(39^{\circ} 40^{\prime} 33.06^{\prime \prime} \mathrm{N}, 72^{\circ} 53^{\prime} 02.06^{\prime \prime} \mathrm{E}\right.$ ) (fig. 1). Furthermore, dog faeces were sampled and tested for E. multilocularis in the same area, and one of them was used to compare genetic profiles. Those genotypic profiles were then compared to other E. multilocularis isolates from Eurasia and North America.

\section{Materials and methods}

In May 2012, a total of 42 Ellobius specimens were trapped within the periphery of Sary Mogol village using tong traps, in an area of about 0.53 ha $\left(72^{\circ} 53^{\prime} 27.78^{\prime \prime} \mathrm{E}\right.$, $39^{\circ} 40^{\prime} 50.952^{\prime \prime} \mathrm{N}$ ) at an altitude of $3000 \mathrm{~m}$. As in every other household of this area, the hamlet was surrounded by Alpine grassland and farmland (fig. 2a). Eastern mole voles were identified to the specific level using conspicuous and typical morphometric criteria (short and soft fur, small eyes, long and straight incisors extending far forward of the nasal cavities; fig. 2c). All animals were weighed, measured and sexed in a field laboratory. Rodent eyeballs were collected to assess their relative age by using their dry crystalline weight, and were preserved in 5\% formalin (Kozakiewicz, 1976). At necropsy, the liver and lungs were examined macroscopically for any lesions. When lesions were found, samples were collected and stored in a 90\% alcohol solution. The presence of protoscoleces was assessed under microscopy after a puncture into the lesion with a syringe. Rodent carcases were preserved in $10 \%$ formalin for reference collection.

Dog faeces were sampled in Sary Mogol and other villages over the same period. Echinococcus multilocularis DNA was amplified from dog faeces found in Taldy Suu village $\left(72^{\circ} 58^{\prime} 15.75^{\prime \prime} \mathrm{E}, 39^{\circ} 42^{\prime} 24.41^{\prime \prime} \mathrm{N}\right)$ situated $7.4 \mathrm{~km}$ from the small mammal sampling spot (see van Kesteren et al., 2013).

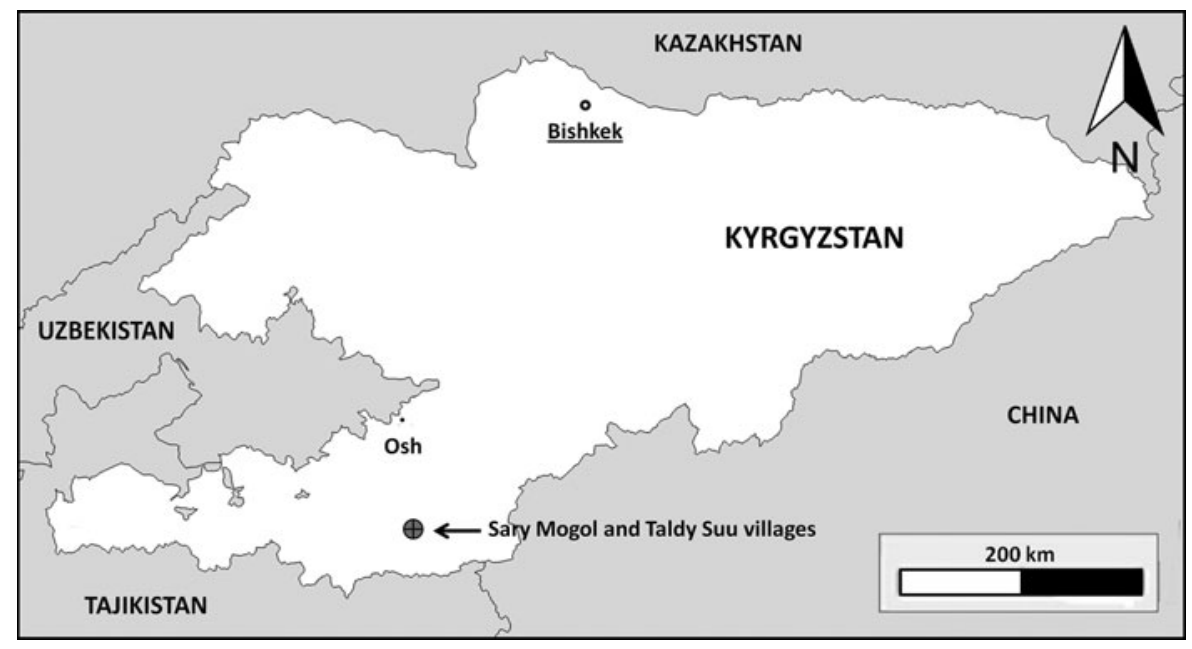

Fig. 1. Map of Kyrgyzstan to show the study site (circled) in the Alay valley. 

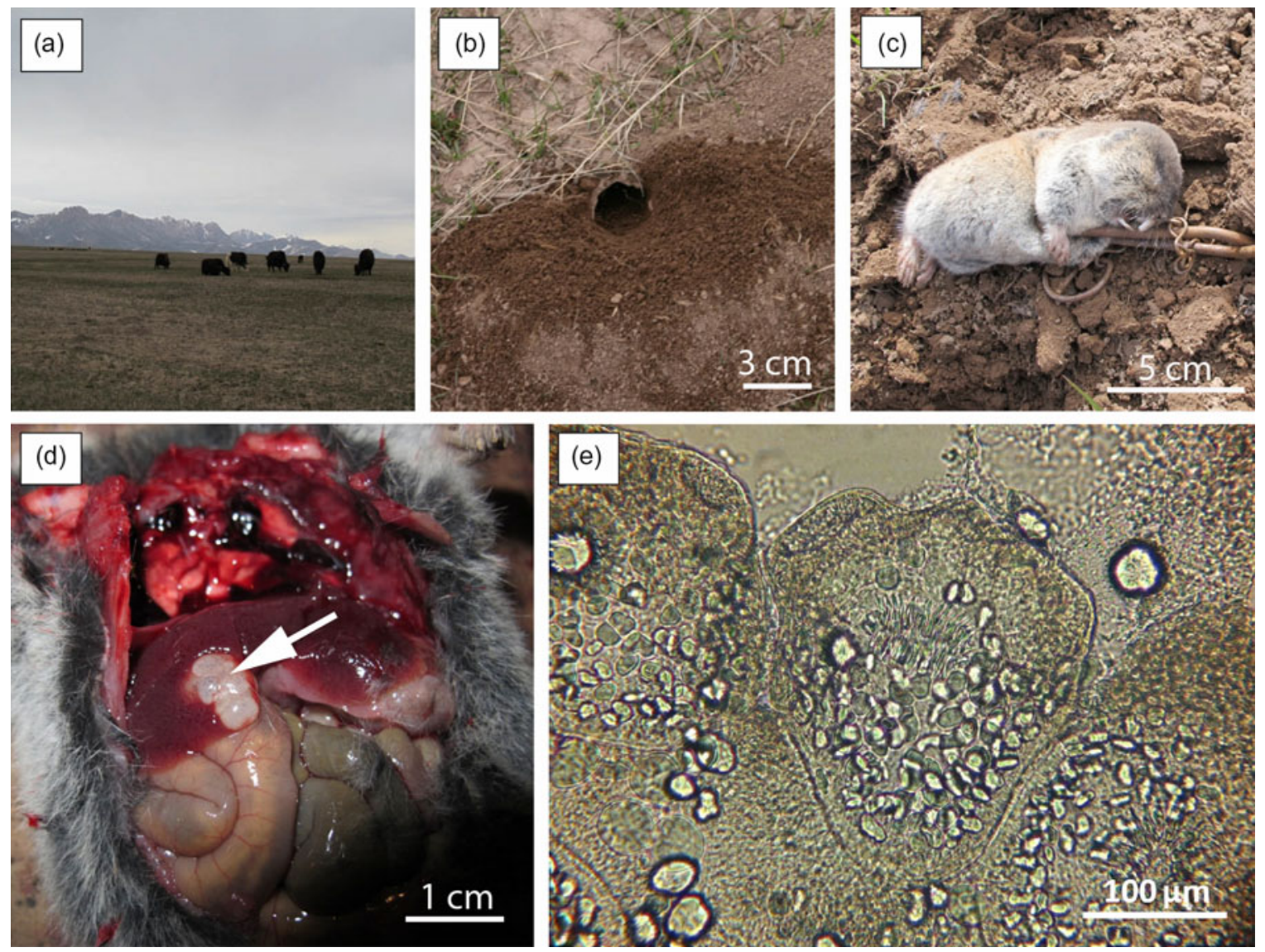

Fig. 2. (a) The landscape of the Alay valley with (b) a burrow entrance of Ellobius tancrei; (c) an entire specimen of E. tancrei caught in a tong trap; (d) liver lesion (arrowed) caused by Echinococcus multilocularis; (e) invaginated protoscolex of E. multilocularis.

Total genomic DNA from the rodent liver lesion was extracted by using the High Pure PCR Template Preparation kit (Roche Diagnostics, Mannheim, Germany), as recommended by the manufacturer. The Echinococcus species determination was done with DNA amplification by polymerase chain reaction (PCR) and sequencing of the mitochondrial DNA (mtDNA) fragment of the $n d 1$ gene (primers ND1_Fwd: 5'-AGATTCGTAAGGGGCCTAATA-3' and ND1_Rev: 5'-ACCACTAACTAATTCACTTTC-3'; Bowles \& McManus, 1993) and compared to the GenBank database. Sequencing using the Sanger method was performed from the two ND1 primers, in order to obtain a consensus sequence. For the dog faecal sample, DNA was extracted using a Qiagen stool mini kit (Qiagen, Hilden, Germany) following the manufacturer's instructions but using $1 \mathrm{~g}$ of faeces. The positive dog faecal sample from Taldy Suu was also amplified for the $n d 1$ gene.

Genotyping of parasite samples was performed by amplification of the tandemly repeated microsatellite EmsB as described previously (Knapp et al., 2007) and modified (Umhang et al., 2014). Briefly, the reaction was performed in a $25 \mu \mathrm{l}$ reaction mixture, containing $200 \mu \mathrm{M}$ of each deoxynucleoside triphosphate (dNTP), $0.4 \mu \mathrm{M}$ fluorescent forward primer EmsB A (5'FAM-GTGTGGATGAGTGTGCCATC-3'), $0.7 \mu \mathrm{M}$ classical reverse primer EmsB C (5'-CCACCTTCCCTACTGCAATC-3') and $0.5 \mathrm{U}$ of Platinum Taq DNA polymerase enzyme (Life Technologies, Foster City, California, USA), with the addition of Platinum $1 \times$ PCR buffer (Life Technologies). The amplification reaction was performed in a Veriti thermocycler (Life Technologies), under the following conditions: a pre-amplification step of $94^{\circ} \mathrm{C}$ for $2 \mathrm{~min}$; followed by 45 cycles with a denaturing step at $94^{\circ} \mathrm{C}$ for $30 \mathrm{~s}$, annealing at $60^{\circ} \mathrm{C}$ for $30 \mathrm{~s}$ and extension at $72^{\circ} \mathrm{C}$ for $1 \mathrm{~min}$; with a final elongation at $72^{\circ} \mathrm{C}$ for $45 \mathrm{~min}$. The PCR products were analysed by fragment size analysis using an ABI Prism 310 apparatus and the GeneMapper 4.1 software (Life Technologies, Carlsbad, California, USA). The Kyrgyz sample isolated from $E$. tancrei was compared to a database composed of 1084 genotyped samples from Europe (France, $n=537$; Germany, $n=88$; Switzerland, $n=109$; Austria, $n=99$; Slovakia, $n=63$; Czech Republic, $n=66$; and Poland, $n=94$ ), from Asia (Tibetan plateau in China, $n=5$; Hokkaido in Japan, $n=6$ ) and from North America (Canada, $n=1$; Alaska, $n=13$ ). The Kyrgyz positive dog faecal sample contaminated by E. multilocularis $(n=1)$ was included, and a sample of E. granulosus sensu stricto as 
an outgroup $(n=2)$. The genetic distance amongst samples was assessed by Euclidean distance between EmsB profiles. As described previously, two samples were considered as identical when the genetic distance was below 0.08 (Knapp et al., 2007).

\section{Results}

Among the 42 individuals, 15 were females and 27 males. The body weight ranged from 47 to $77 \mathrm{~g}$ and crystalline dry mass from 0.45 to $3.8 \mathrm{mg}$. One Ellobius specimen, an adult male, was caught by hand and brought by children from the hamlet. Its body weight was $62 \mathrm{~g}$ and crystalline dry mass $1.1 \mathrm{mg}$. This specimen was the only individual that presented larval cysts of E. multilocularis. It showed two liver lesions $(12-18 \mathrm{~mm}$ in diameter; fig. 2d). Protoscoleces were found after examining cyst vesicle fluid under a light microscope (fig. 2e). For both the Ellobius specimen and the dog faecal sample, the amplification of the mtDNA fragment of the $n d 1$ gene allowed us to generate a 400-bp consensus sequence. The two isolates had $100 \%$ identity with each other and presented $99 \%$ identity with the nd1 sequence from the complete mitochondrial genome (AB018440.2). One mutation was observed (position 8012 G/A mutation) in the referenced sequence in both the forward and reverse sequences, in comparison to the other sequences referenced in the GenBank database for the E. tancrei sample and the dog faeces extract (see sequences in fig. 3). This mutation was observed amongst, for example, a Polish sample (GenBank reference: AJ132908.1) and Chinese samples (Xinjiang sample: EU704124.1 and Sichuan: EU704123.1), these reference samples having the nucleotide $\mathrm{A}$ at the position 8012 in the nd1 gene, and the Kyrgyz samples a nucleotide G. The presence of the mutation was confirmed by performing the sequencing twice. In comparison to the EmsB database $(n=1084$ samples) no identical samples $(<0.08$ of genetic distance) were clustered with the Kyrgyz sequences (from E. tancrei and the dog faecal samples), but the two Kyrgyz sequences were clustered together with a genetic distance of 0.12. They can subsequently been considered as similar strains but not identical, perhaps due to poor DNA quality (fig. 4). Moreover, the two samples were linked with Tibetan (China) and Hokkaido (Japan) samples, and one Alaskan sample, with a genetic distance ranging from 0.17 to 0.24 (fig. 4), but with neither the European nor American isolates.

\section{Discussion}

The current results add further information about the natural infection of the Eastern vole mole, E. tancrei, with E. multilocularis, first discovered more than 50 years ago. These findings based on EmsB genotyping indicate, first, that the two isolates (vole and dog) found in our study belong to the Asian strain of E. multilocularis, hence extending the western limit of the known distribution range of this genotype in central Asia. The Pamir Mountain range is situated in altitudinal continuity with the Tibetan plateau but, due to its complex high-altitude ranges, might have been considered a biogeographical barrier to the spread of the eastern Asian strain of E. multilocularis to the central Asian republics - a hypothesis that is refuted here. Second, very similar strains were found in dog faeces and the E. tancrei specimen in the study area, and the common mutation first described in the present study emphasized, as a fingerprint, the involvement of E. tancrei and dogs in the local parasite cycle. The occurrence of this mutation amongst Asian E. multilocularis isolates needs further studies to be understood. Associated with the fact that E. tancrei could be trapped at less than $10 \mathrm{~m}$ from house walls, and all of them at less than $100 \mathrm{~m}$, this indicates that a synanthropic

\begin{abstract}
$>$ E.multilocularis_ND1_Ellobius tancrei
GGTTTGTTGGGCGTTTTTTTGTTAATAATTTTGGTTATTATATATTCTTTTATTTATGGTAGATATTATAGTGTTAGTTA TAATAGTCTTTCAGTATTGTGGTTTTTAGCTGTTGCTAGTATTTCTAGGTATTCTTTGTTGTGTGCTGGTTGGGGTA GTTACAATAAATATTCGTTTTTAAGTTCTGTTCGATGTGCTTTTGGGTCTGTTAGGTTTGAAGCTTGTTTTATGTGT GTGGTAATTTTTTGTTCTTTGTGTTACTGTAGGTATAATTTGATTGATTTTTATTATAGTTGTTGATGGAGCTTGTTG TTGTTTCCATTGATTTATGGATTGTTTTTGGTGTGTGTGCTATGTGAGACTAATCGTATACCATTTGATTATGGGGA GTCTGAAAGTGAA
\end{abstract}

$>$ E. multilocularis_ND1_dog faeces

GGTTTGTTGGGCGTTTTTTTGTTAATAATTTTGGTTATTATATATTCTTTTATTTATGGTAGATATTATAGTGTTAGTTA TAATAGTCTTTCAGTATTGTGGTTTTTAGCTGTTGCTAGTATTTCTAGGTATTCTTTGTTGTGTGCTGGTTGGGGTA GTTACAATAAATATTCGTTTTTAAGTTCTGTTCGATGTGCTTTTGGGTCTGTTAGGTTTGAAGCTTGTTTTATGTGT GTGGTAATTTTTTGTTCTTTGTGTTACTGTAGGTATAATTTGATTGATTTTTATTATAGTTGTTGATGGAGCTTGTTG TTGTTTCCATTGATTTATGGATTGTTTTTGGTGTGTGTGCTATGTGAGACTAATCGTATACCATTTGATTATGGGGA

G

Fig. 3. Part of the $n d 1$ gene sequenced from the Ellobius tancrei liver lesion and from the positive dog faecal sample contaminated by Echinococcus multilocularis. The underlined nucleotide corresponds to the mutation position in comparison to the AB018440.2 complete mitochondrial genome referenced. 
Fragment size (base pair)

Genetic distance

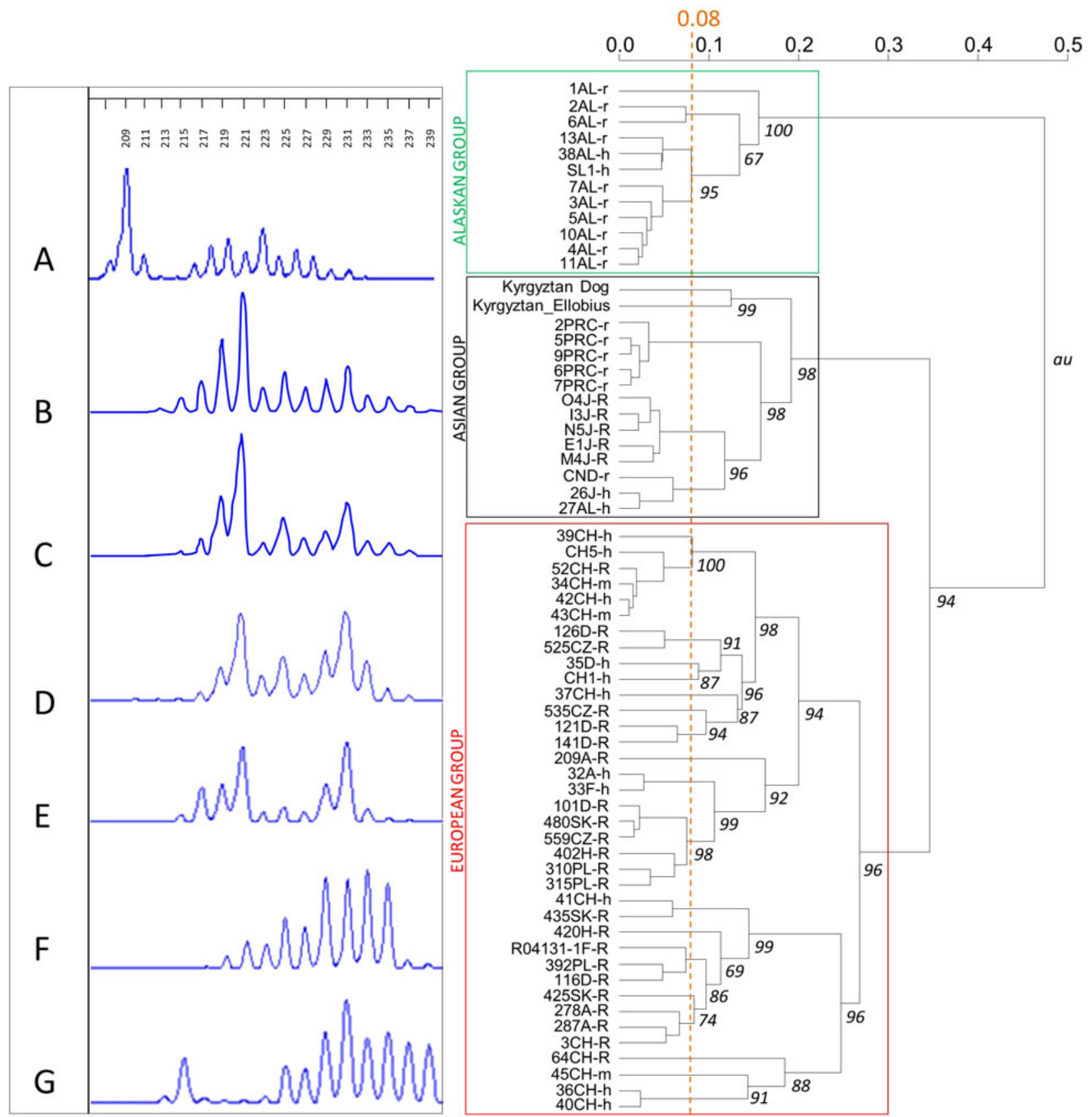

Fig. 4. EmsB profiles of Echinococcus multilocularis from samples in (A) Alaska; (B) dog faeces, Kyrgyzstan; (C) liver of Ellobius tancrei, Kyrgyztan; (D) liver of Microtus limnophilus, Siqhu, Tibetan plateau, Sichuan, China; (E) fox intestine, Hokkaido, Japan; and (F, G) fox intestine, Europe. The dendrogram represents the similarities between samples, with bootstrap values $(B=1000)$ at each node and the limit of high similarity being 0.08 (Knapp et al., 2007).

cycle involving dogs and the Eastern mole vole may exist, not excluding the contribution of other small mammal potential host species (e.g. M. gregalis, C. migratorius) that were also observed not only in habitats remote from villages but also in the close vicinity of houses, where Mus musculus was also captured. Large population densities of both dogs and E. tancrei were observed in the Alay valley. Ellobius tancrei abundance has been shown to increase with grassland vegetation biomass (Giraudoux et al., 2013). This leads to the maintenance of larger vole populations in farmland that surrounds villages, where barley is grown, and in hay fields close to villages, with 
vole population spillover into villages. Moreover, 38-74\% of households have at least one dog in the villages studied in the Alay Valley (van Kesteren et al., 2013), which leads to a high concentration of potentially infective dog faeces. This should be added to a large red fox population in the area, with tens of fox dens found at less than $1-2 \mathrm{~km}$ from villages (Giraudoux and Rieffel, pers. obs.), which may also feed the sustainable transmission of E. multilocularis (however, see Liccioli et al., 2015). Third, the only specimen of $E$. tancrei found to be infected by $E$. multilocularis was also the only specimen caught by hand by children. This might indicate that the animal found infected in the present study might have been caught not by chance but as the result of an increased vulnerability to capture induced by the parasite. This possibly altered host-behavioural aspect of the transmission ecology of E. multilocularis appears not to have been mentioned previously in the literature, and should be investigated carefully, using appropriate methods.

\section{Acknowledgements}

We thank Alexander Mastin, Mike Rogan and Bermet Mytynova for their help in obtaining the domestic dog sample.

\section{Financial support}

Financial support was received from the Wellcome Trust (\#094325/Z/10/Z programme). This research has been conducted within the context of the GDRI (International research network) 'Ecosystem health and environmental disease ecology'.

\section{Conflict of interest}

None.

\section{References}

Bart, J.M, Knapp, J., Gottstein, B., El-Garch, F., Giraudoux, P., Glowatzki, M.L., Berthoud, H., Maillard, S. \& Piarroux, R. (2006) EmsB, a tandem repeated multi-loci microsatellite, new tool to investigate the genetic diversity of Echinococcus multilocularis. Infection Genetics and Evolution 6, 390-400.

Batsaikhan, N. \& Tinnin, D. (2008) Ellobius tancrei. IUCN Red List of Threatened Species, Version 2013.1. Available at http://www.iucnredlist.org (accessed 16 September 2013).

Bowles, J. \& McManus, D.P. (1993) NADH dehydrogenase 1 gene sequences compared for species and strains of the genus Echinococcus. International Journal for Parasitology 23, 969-972.

Bretagne, S., Assouline, B., Vidaud, D., Houin, R. \& Vidaud, V. (1996) Echinococcus multilocularis: microsatellite polymorphism in U1 snRNA genes. Experimental Parasitology 82, 324-328.

Eckert, J. \& Deplazes, P. (2004) Biological, epidemiological, and clinical aspects of echinococcosis, a zoonosis of increasing concern. Clinical Microbiology Reviews 17, 107-135.
Eckert, J., Gemmel, M.A., Meslin, F.-X. \& Pawlowski, Z.S. (Eds) (2001) WHO/OIE manual on echinococcosis in humans and animals: a public health problem of global concern. Paris, Office International des Epizooties.

Gagarin, V.G., Steshenko, V.M. \& Tokobaev, M.M. (1957) Role of rodents in the distribution of helminth zoonoses. Works of the Institute Zoology and Parasitology of the Kyrgyz Academy of Sciences 6, 159-161 (in Russian).

Giraudoux, P., Pleydell, D., Raoul, F., Quere, J.P., Wang, Q., Yang, Y.R., Vuitton, D.A., Qiu, J.M., Yang, W. \& Craig, P.S. (2006) Transmission ecology of Echinococcus multilocularis: what are the ranges of parasite stability among various host communities in China? Parasitology International 55, S237-S246.

Giraudoux, P., Zhou, H., Quéré, J.P., Raoul, F., Delattre, P., Volobouev, V., Déforêt, T., Ito, A., Mamuti, W., Scheifler, R. \& Craig, P.S. (2008) Small mammal assemblages and habitat distribution in the northern Junggar Basin, Xinjiang, China: a pilot survey. Mammalia 72, 309-319.

Giraudoux, P., Raoul, F., Afonso, E., Ziadinov, I., Yang, Y., Li, L., Li, T.Y., Quéré, J.P., Feng, X.H., Wang, Q., Wen, H., Ito, A. \& Craig, P.S. (2013) Transmission ecosystems of Echinococcus multilocularis in China and Central Asia. Parasitology 140, 1655-1666.

Haag, K.L., Zaha, A., Araújo, A.M. \& Gottstein, B. (1997) Reduced genetic variability within coding and noncoding regions of the Echinococcus multilocularis genome. Parasitology 115, 521-529.

Knapp, J., Bart, J.M., Glowatzki, M.L., Ito, A., Gerard, S., Maillard, S., Piarroux, R. \& Gottstein, B. (2007) Assessment of use of microsatellite polymorphism analysis for improving spatial distribution tracking of Echinococcus multilocularis. Journal of Clinical Microbiology 45, 2943-2950.

Knapp, J., Guislain, M.H., Bart, J.M., Raoul, F., Gottstein, B., Giraudoux, P. \& Piarroux, R. (2008) Genetic diversity of Echinococcus multilocularis on a local scale. Infection, Genetics and Evolution 8, 367-373.

Knapp, J., Bart, J.M., Giraudoux, P., Glowatzki, M.L., Breyer, I., Raoul, F., Deplazes, P., Duscher, G., Martinek, K., Dubinsky, P., Guislain, M.H., Cliquet, F., Romig, T., Malczewski, A., Gottstein, B. \& Piarroux, R. (2009) Genetic diversity of the cestode Echinococcus multilocularis in red foxes at a continental scale in Europe. PLoS Neglected Tropical Diseases 3, e452.

Kozakiewicz, M. (1976) The weight of eye lens as the proposed age indicator of the bank vole. Acta Theriologica 21, 314-316.

Liccioli, S., Giraudoux, P., Deplazes, P. \& Massolo, A. (2015) Wilderness in the 'city' revisited: different urbes shape transmission of Echinococcus multilocularis by altering predator and prey communities. Trends in Parasitology pii: S1471-4922(15)00084-7. doi:10.1016/j.pt.2015.04.007 [Epub ahead of print].

Tokobaev, M.M. (1959) Helminths of rodents in Kyrgyzia. Works of the Institute Zoology and Parasitology of the Kyrgyz SSR Academy of Sciences 7, 133-142 (in Russian).

Torgerson, P.R., Oguljahan, B., Muminov, A.E., Karaeva, R.R., Kuttubaev, O.T., Aminjanov, M. \& Shaikenov, B. (2006) Present situation of cystic echinococcosis in Central Asia. Parasitology International 55, 207-212. 
Umhang, G., Knapp, J., Hormaz, V., Raoul, F. \& Boué, F. (2014) Using the genetics of Echinococcus multilocularis to trace the history of expansion from an endemic area. Infection, Genetics and Evolution 22, 142-149.

Usubalieva, J., Minbaeva, G., Ziadinov, I., Deplazes, P. \& Torgerson, P.R. (2013) Human alveolar echinococcosis in Kyrgyzstan. Emerging Infectious Diseases 19, 1095-1097.

van Kesteren, F., Mastin, A., Mytynova, B., Ziadinov, I., Boufana, B., Torgerson, P.R., Rogan, M.T. \& Craig, P.S. (2013) Dog ownership, dog behavior and transmission of Echinococcus spp. in the Alay Valley, southern Kyrgyzstan. Parasitology 140, 1674-1684.

Vuitton, D., Zhou, H., Bresson-Hadni, S., Wang, Q., Piarroux, M., Raoul, F. \& Giraudoux, P. (2003)
Epidemiology of alveolar echinococcosis with particular reference to China and Europe. Parasitology 127, S87-107.

Ziadinov, I., Mathis, A., Trachsel, D., Rysmukhambetova, A., Abdyjaparov, T.A., Kuttubaev, O.T., Deplazes, P. \& Torgerson, P.R. (2008) Canine echinococcosis in Kyrgyzstan: using prevalence data adjusted for measurement error to develop transmission dynamics models. International Journal for Parasitology 38, 1179-1190.

Ziadinov, I., Deplazes, P., Mathis, A., Mutunova, B., Abdykerimov, K., Nurgaziev, R. \& Torgerson, P.R. (2010) Frequency distribution of Echinococcus multilocularis and other helminths of foxes in Kyrgyzstan. Veterinary Parasitology 171, 286-292. 